\title{
Chemical Signals are Involved in the Detection and Preference of Food Sources in Blattella Germanica
}

\author{
Xoana Magalí Santa Cruz a, Valeria Sfara ${ }^{a^{*}}$ \\ ${ }^{a}$ Laboratorio de Control de Plagas de Bajo Impacto Ambiental, Instituto de Investigación e Ingeniería Ambiental, Universidad Nacional \\ de San Martín (3iA - UNSAM), Av. 25 de Mayo y Francia, San Martín (1636) Provincia de Buenos Aires, Argentina
}

\begin{abstract}
The German cockroach (Blattella germanica) is a cosmopolitan omnivorous species which can take nutrients from a wide variety of sources. Its condition of pest generated interest in the study of its biology, and while there are many works regarding its feeding behavior, few approaches have been made to the relevance of the chemosensory system during foraging. Objective: In this work we studied the role of chemical stimuli in the detection and preference of food sources in B. germanica using behavioral observations. Methods: Adult males were placed in a circular experimental arena where different diet types were presented individually. Parameters associated to the detection of odors (latency to the food source, number of visits to the source) were measured in an experimental time of 15 minutes. We also measured the total amount ingested determining the weight gain of each individual, as an indicator of taste evaluation of food. Findings: Insects showed a lower latency when the source emitted a larger amount of volatile compounds, so peanut butter was detected faster compared with the other diets studied. Regarding ingestion, insects spent significantly more time on the peanut butter, indicating the presence of phagostimulants such as sugars or lipids. When sugar solutions were used as food sources, the number of visits, the time spent on the source and the amount consumed was higher when increasing sugar concentration. Insects also showed preference for the solution of higher concentration, when two sugar solutions were presented simultaneously. Latency increased and the amount of sugar ingested decreased when insects' mouthparts were chemically blocked with N-Ethylmaleimide, a general inhibitor of chemoreception. Novelty/Improvement: These results describe how cockroaches use odors to locate food sources and chemoreceptors in their mouthparts to identify diets of higher energetic value, from information regarding the concentration and quality of the food source. Understanding the feeding behavior of cockroaches allows the design of more attractive and palatable toxic gel baits enhancing their efficacy for cockroach control.
\end{abstract}

\section{Keywords:}

Blattella;

Feeding;

Chemoreception;

Odors;

Phagostimulants.
Article History:

Received: 06 August 2018

Accepted: 11 October 2018

\section{1- Introduction}

In nature, animals use stimuli of different sensory modalities coming from potential food sources to locate them from a distance. Visual, thermal and chemical information is used to reach the food source. Chemical information is widely used by insects. Volatile compounds emitted by food are used as odor cues to locate the source from a median/long distance. Then, the chemical composition of the diet is sensed using chemoreceptors present in the mouthparts providing information about the nutritional value of the diet, and helping the insect to decide whether to consume the food or continue searching another one [1][2].

The German cockroach Blattella germanica is a cosmopolitan omnivorous insect, distributed worldwide as a pest. Many studies regarding the biology of feeding in this species can be found in literature. Cockroaches feed on a wide variety of food types and their preference depends on intrinsic and extrinsic variables, such as nutritional state, developmental instar, ovulation cycle in females, age, distance of the source from the refuge, etc. [3].

Regarding attraction of cockroaches to food odors, classic studies from Sugawara et al. [4], and Wileto and Boush [5], compared attractiveness of different odors by observing the number of cockroaches present in adhesive traps loaded

\footnotetext{
*CONTACT: Sfaravaleria@gmail.com

DOI: http://dx.doi.org/10.28991/esj-2018-01150

(C) This is an open access article under the CC-BY license (https://creativecommons.org/licenses/by/4.0/).
} 
with different volatile compounds, after several hours of exposure. Authors discussed the difference in attractiveness in relation to the chemical structure of the attractant, but, although interesting, these works are mainly descriptive and make no reference to the role of the sensory system in this process. More recently, Lauprasert et al. [3] showed that cockroaches were more attracted to diets containing carbohydrates such as bread, sugar, banana, and potato, and observed a similar preference pattern between males and females. Regarding the role of taste in the decision of exploiting a food source, the information available is scarce. These studies focused on the function of taste receptors in the glucoseaverse strain of B. germanica [6-8].

In this work we used different diet types (peanut butter, dehydrated yeast and sugar solutions) which differ in chemical composition and in volatile emissions, in order to describe the feeding behavior of B. germanica associated with olfaction and taste. German cockroaches are efficiently reared in laboratory feeding on peanut butter and dehydrated yeast (unpublished data). Peanut butter is a paste composed by peanut and honey. Volatile compounds expected to be emitted by peanut butter can be grouped into chemical categories such as aldehyde, ketone, acid, alcohol, hydrocarbon, norisoprenoids, terpenes and benzene compounds, furan and pyran derivatives, which are the major compounds emitted by peanut and honey $[9,10]$.

We defined two main macro-variables to describe the feeding behavior of B. germanica: latency, related with the reception of volatile compounds and the function of olfaction, and weight gain, related to the perception of phagostimulants and the function of taste. Phagostimulants are chemicals present in food that favor its ingestion [11]. The aim of this work was to determine by the observation of behavior at short experimental times, the role of chemical stimuli in the orientation towards a food source and in the decision-making mechanism for its exploitation, in the German cockroach B. germanica.

\section{Materials and Methods}

\section{2-1- Biological Material}

Males of B. germanica from a colony reared in laboratory in controlled conditions of temperature, humidity, and photoperiod $\left(24{ }^{\circ} \mathrm{C} ; 60 \%\right.$ humidity; $12: 12$ light-dark cycle) were used. Insects were fed with rabbit pellet and water ad libitum. Male cockroaches were separated and kept unfed with water ad libitum for $2 / 3$ hours prior to the experiments.

\section{2-2- Chemicals}

Solutions of sucrose or fructose in distilled water were used as sugar sources (2, 0.2 and $0.02 \mathrm{~g} / \mathrm{ml})$. Sucrose and fructose were donated by Chemotecnica S.A. (Ezeiza, Buenos Aires, Argentina).

N-Ethylmaleimide (NEM) was provided by Sigma (Darmstadt, Germany). A solution of $10 \mathrm{mg} / \mathrm{ml}$ of NEM in ethanol was used as chemoreception blocker.

\section{2-3- Experimental Device}

The experimental arena consisted of a circular acrylic container $(18 \mathrm{~cm}$ of diameter and $20 \mathrm{~cm}$ of height), with its internal base covered with filter paper, which was replaced in each replicate.

A known amount of each food source was weighed into a circular glass container $(3 \mathrm{~cm}$ in diameter $)$ and then placed in the center of the arena. One insect was gently released in it and its behavior was observed. Each diet was offered individually.

\section{2-4- Bioassays}

$10 \mathrm{mg}$ of each solid diet was offered individually. One male was placed in the arena and the following variables were measured during 15 minutes:

- Latency - time in which the insect reaches the food source for the first time

- Number of visits

- Weight gain $(\mathrm{mg})$ - determined as the difference in the weight of the insect before and after the experiment.

Different types of foods were used:

Dehydrated yeast (DY); Dehydrated yeast with the addition of water (HY); Peanut butter (PB).

Each diet varied in their composition and in the amount of volatiles emitted. Briefly, DY is rich in proteins, as well as HY. The addition of water to the DY increases the release of volatile compounds, such as alcohols and $\mathrm{CO}_{2}$. On the other hand, PB is rich in carbohydrates and lipids, and emits a large amount of volatiles associated with the two major ingredients, peanut and honey.

In order to describe the feeding behavior of insects offered with a food source composed by a single ingredient, solutions of sucrose and fructose were used. Solutions were prepared in distilled water in three different concentrations: 
high sugar $(\mathrm{HS}=2 \mathrm{~g} / \mathrm{ml})$; middle sugar $(\mathrm{MS}=0.2 \mathrm{~g} / \mathrm{ml})$; low sugar $(\mathrm{LS}=0.02 \mathrm{~g} / \mathrm{ml})$.

$1 \mathrm{ml}$ of each solution was offered individually to a single B. germanica male, and the variables stated above were determined. Control group was offered with $1 \mathrm{ml}$ of distilled water.

In order to study the role of olfaction in the feeding behavior of cockroaches, another experimental series was preformed, offering sugar solutions adding to the center of the arena a filter paper treated with banana smell as an attractant. For this, a piece of banana peel was rubbed on the filter paper to aromatize it.

20 independent replicates of each experiment were performed.

\section{2-5- Preference among Sugar Solutions}

Two solutions of sucrose or fructose of different concentrations were presented simultaneously, to study the ability of cockroaches to choose food sources by its concentration. Three pairs of sugar solutions were composed: HS-MS; HSLS; MS-LS. A male cockroach was placed in the arena and the time spent feeding on each of the solutions was determined. A Preference Index (PI) was calculated with the data obtained. The construction of the PI is explained in the Data Analysis section.

An additional experiment was conducted to determine if the insects were able to differentiate the sugar type regardless of their concentration. Two high sugar solutions $(2 \mathrm{~g} / \mathrm{ml})$, one of sucrose and one of fructose, were simultaneously offered to B. germanica males, and preference was determined as described above. 20 independent replicates of each experiment were performed.

\section{2-6- Chemoreception Blocking}

To confirm the role of chemoreceptors in the evaluation of food quality, $1 \mu 1$ of a solution of NEM $(10 \mathrm{mg} / \mathrm{ml}$ in ethanol) was applied to a B. germanica male in the mouthparts using a microsyringe provided with a dispenser (Hamilton Company, Reno, USA). Immediately after treatment, insects were placed in the experimental arena where a HS solution of sucrose was offered and the behavioral parameters were determined as previously described. A preference experiment was performed exposing treated males to a sucrose vs fructose choice trial. Control group was treated with $1 \mu \mathrm{l}$ of ethanol alone. An additional control with untreated insects was performed in each experiment. 15 independent replicates were conducted.

\section{2-7- Data Analysis}

Latency time, number of visits and weight gain were compared using non-parametrics Kruskall-Wallis and Dunn's test for post-hoc comparisons when required.

To assess the preference between sugar solutions, the Preference Index (PI) was calculated as follows:

Preference index (PI): Ti - Tip / Ti

Where:

Ti is the effective time of ingestion, which is the time that insects spend eating on any of the diets, from the total experimental time. Tip is the time of ingestion on the solution of lower concentration of the pair.

PI varies from 0 to 1 . PI $=0$ indicates preference for the less concentrated solution of the pair, while PI $=1$ indicates preference for the more concentrated one. $\mathrm{PI}=0.5$ indicates random choice of any diet.

To determine if the PIs were significantly different from 0.5 , the Student's t test for a parameter was used. A transformation of the data was applied when it was necessary.

\section{3- Results}

In order to describe parameters of the feeding behavior of cockroaches, three solid diets of different composition were offered, and latency, number of visits to the source, and weight gain were determined. Cockroaches found the peanut butter significantly faster than the other foods offered ( $p<0.05$, Kruskall-Wallis and Dunn's test). Regarding yeast, the latency was shorter when yeast was added with water. These results are shown in Figure 1a. During the experimental time, insects made less visits to the peanut butter compared to yeast sources ( $p<0.05$, Kruskall-Wallis and Dunn's test; Figure 1b). However, no statistically significant differences were observed in weight gain among diets ( $p>0.05$, Kruskall-Wallis; Figure 1c). 

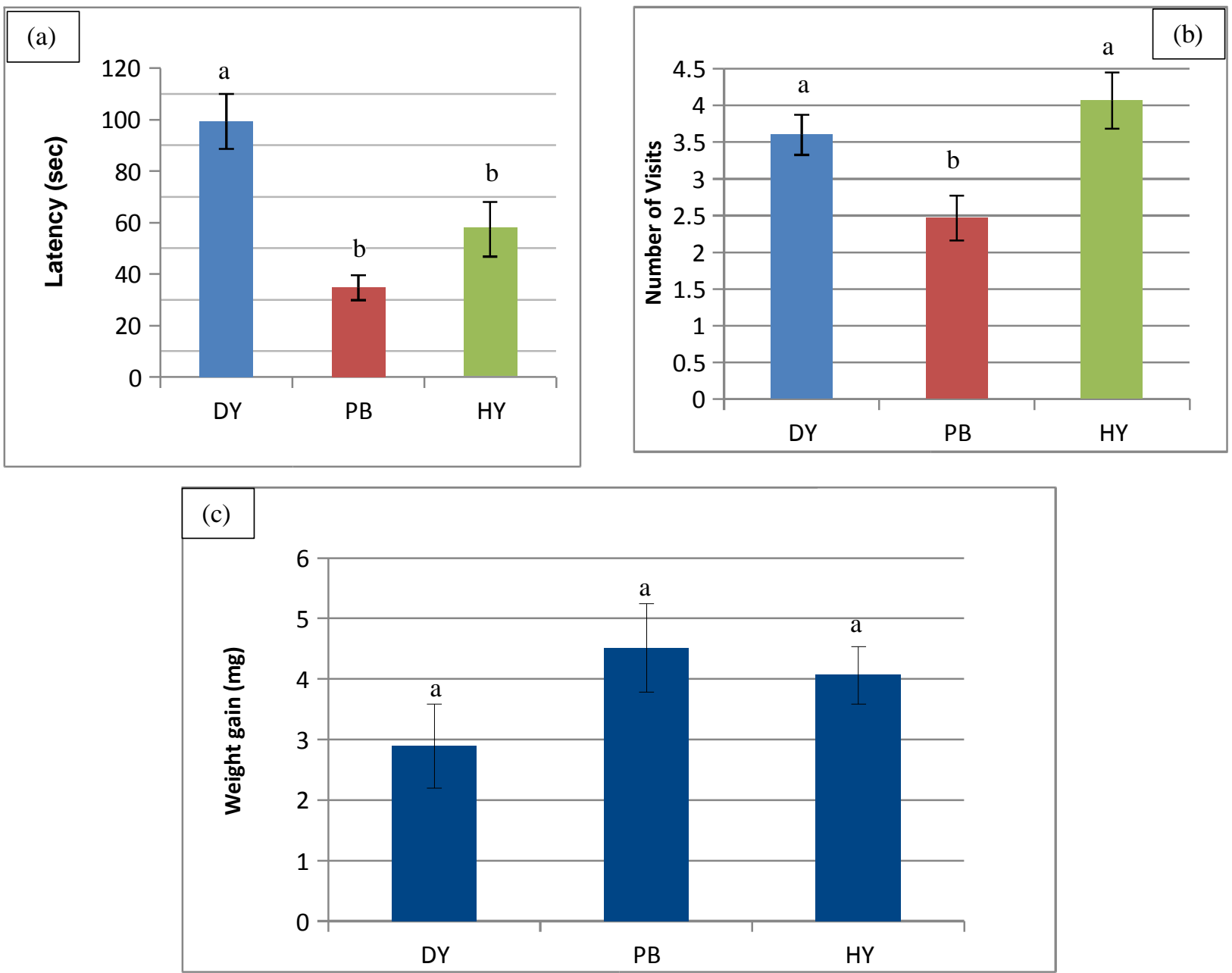

Figure 1. Feeding performance of $B$. germanica with solid diets (DY = dehydrated yeast; $\mathbf{P B}=$ peanut butter; $\mathbf{H Y}=$ dehydrated yeast + water). Three parameters were observed: a) Latency; b) Number of visits to the source; c) Weight gain. Different letters indicate statistically significant differences.

The performance of cockroaches in the consumption of simple diets was studied using sucrose solutions of different concentrations. The same parameters as for solid diets were determined. A lower latency was observed as the concentration of sucrose increased, although sugar solutions do not emit volatiles due to their low vapor pressure. The role of odors in the detection of food sources from a median distance was confirmed adding banana smell to the experimental arena. The same pattern was observed in which latency to sucrose solutions was significantly lower as the concentration increased, but it was even lower when the arena was scented with banana ( $p<0.05$, Kruskall-Wallis and Dunn's test; Figure 2a).

The number of visits to the sugar sources was enhanced by the presence of banana odor. In all the concentrations of sugar tested, the mean number of visits was significantly higher in the presence of banana smell ( $p<0.05$, KruskallWallis and Dunn's test; Figure 2b). Conversely, the presence of an odor did not affect the total amount ingested. The weight gain was significantly higher as sugar concentration increased, and the addition of banana smell did not modify this pattern ( $\mathrm{p}<0.05$, Kruskall-Wallis and Dunn's test; Figure 2c). 

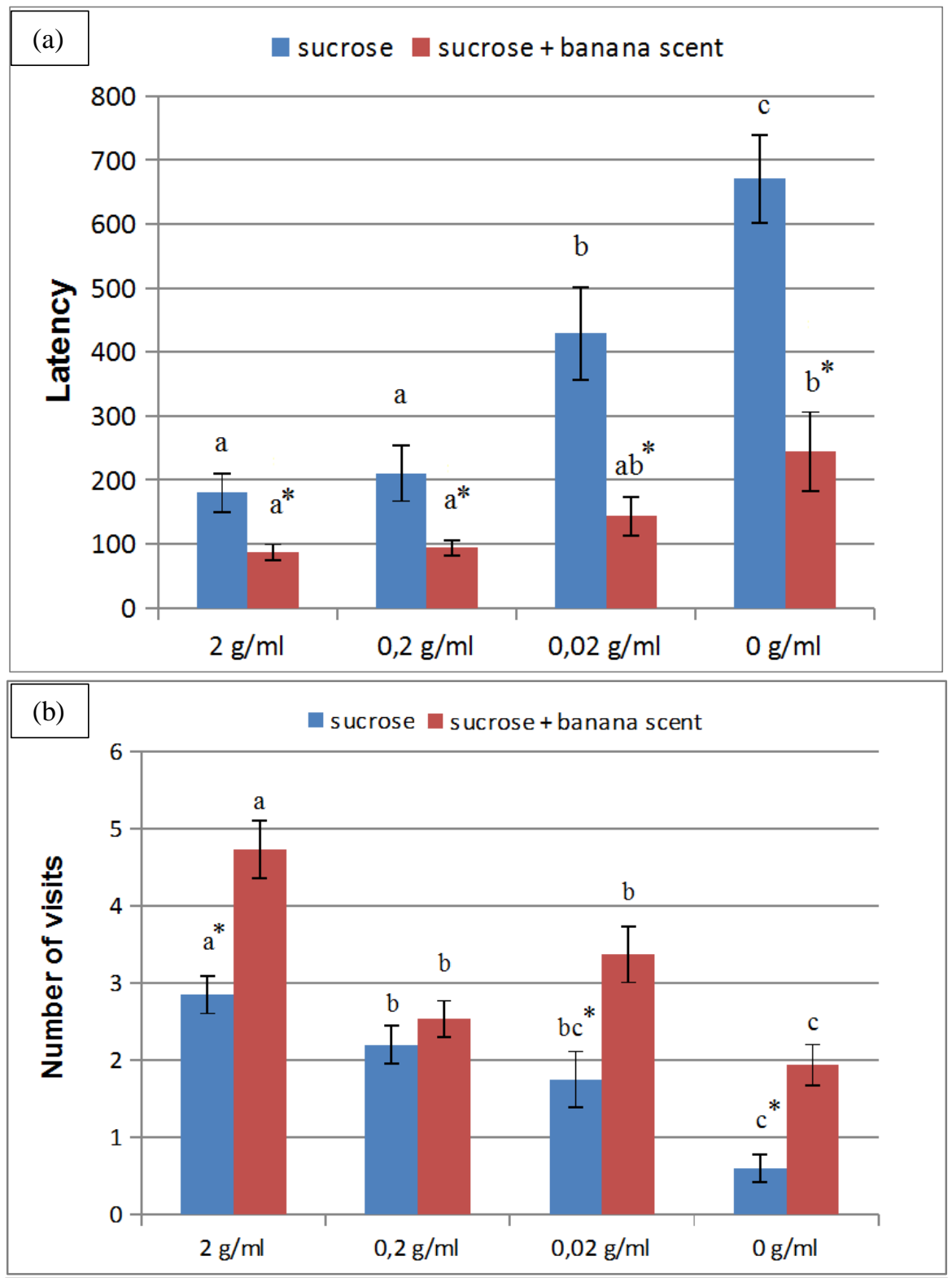

(c) $\quad$ sucrose $\quad$ sucrose + banana scent

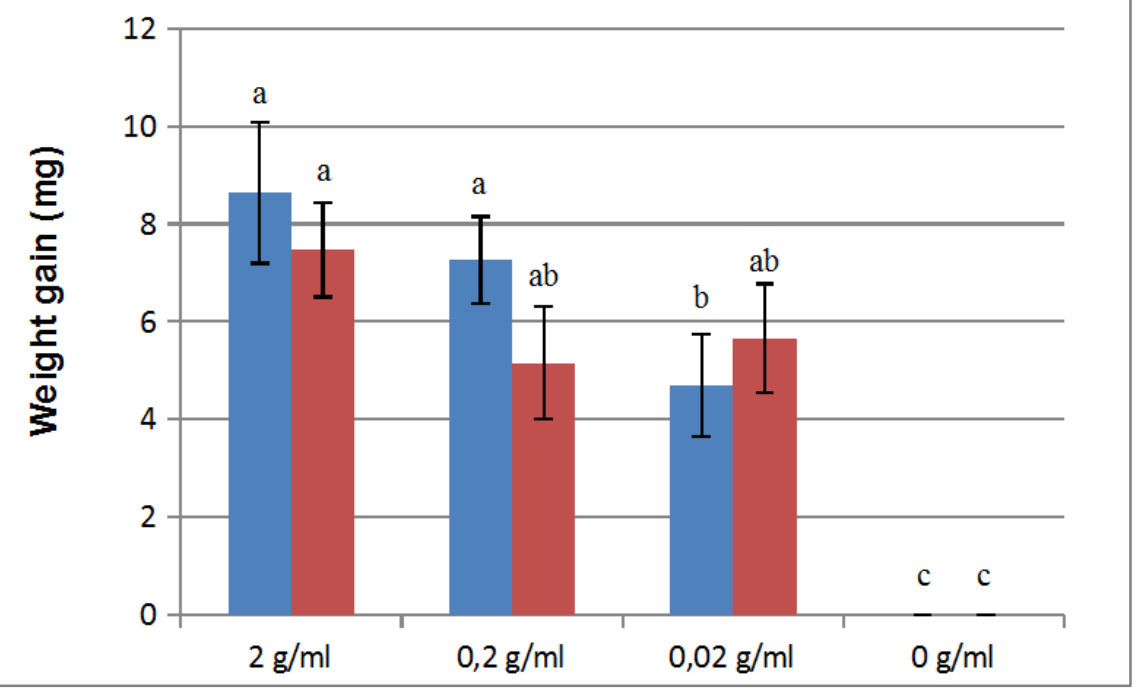

Figure 2. Feeding performance of $\boldsymbol{B}$. germanica with sucrose solutions in distilled water. The following parameters were analyzed: a) Latency; b) Number of visits to the source; c) Weight gain. Different letters indicate statistically significant differences among treatments; marks indicate statistically significant differences between bars. 
When preference for sucrose solutions of different concentrations was tested, insects always choose the most concentrated of the pair. Figure 3 shows the PIs for the three combinations of sugar solutions used (HS-MS; HS-LS; MS-LS). In all cases, PIs were significantly different from 0.5 and near 1 , indicating the preference for the solution of higher sugar of the pair ( $\mathrm{p}<0.05$, Student's t test for a parameter).

Similar results were obtained when solutions of fructose were used. As in the case of sucrose, when different concentrations of fructose were presented simultaneously, insects always preferred the higher sugar solution of the pair. PIs were also significantly different from 0.5 and near 1 ( $\mathrm{p}<0.05$, Student's t test for a parameter; Figure 4).

An additional result is presented in Figure 4, which shows the ability of cockroaches to identify the type of sugar in addition to its concentration. Two solutions of $2 \mathrm{~g} / \mathrm{ml}$, one of sucrose and one of fructose, were presented simultaneously and a significant preference for fructose was observed (IP $=0.73 \pm 0.060$, considering Tip as the time spent on the sucrose source). The IP was significantly different from 0.5 ( $\mathrm{p}<0.05$, Student's t test for a parameter).

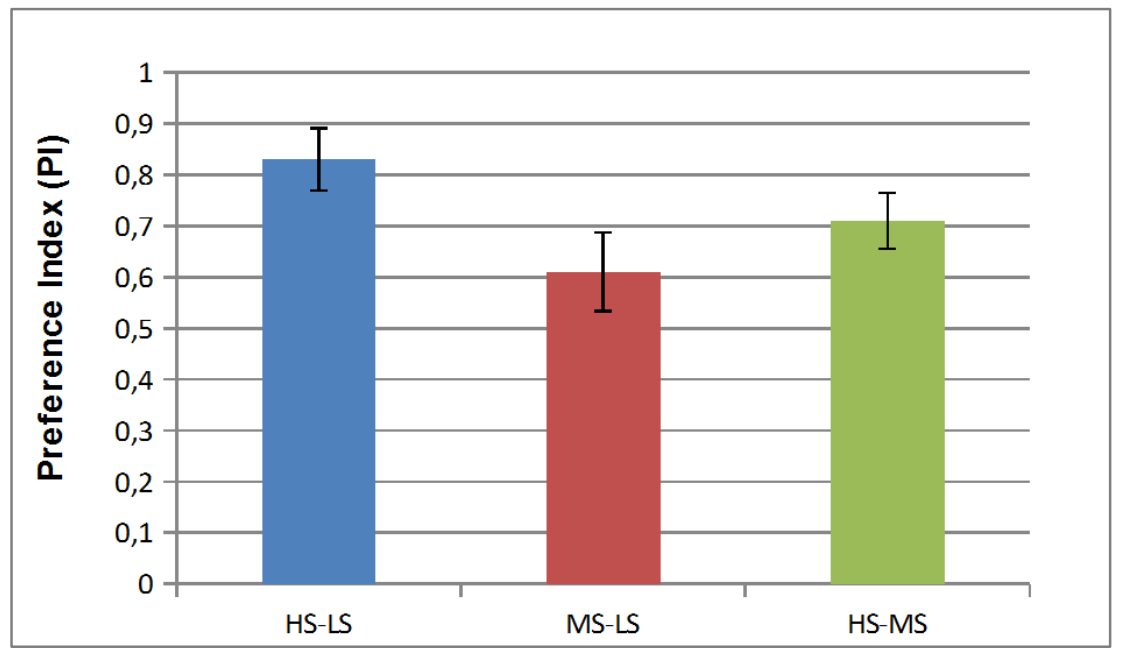

Figure 3. Preference Index (PI) of B. germanica exposed to pairs of sucrose solutions of different concentration (HS = 2 $\mathrm{g} / \mathrm{ml} ; \mathrm{MS}=0.2 \mathrm{~g} / \mathrm{ml} ; \mathrm{LS}=0.02 \mathrm{~g} / \mathrm{ml})$. PIs near 1 indicate preference for the most concentrated solution of the pair.

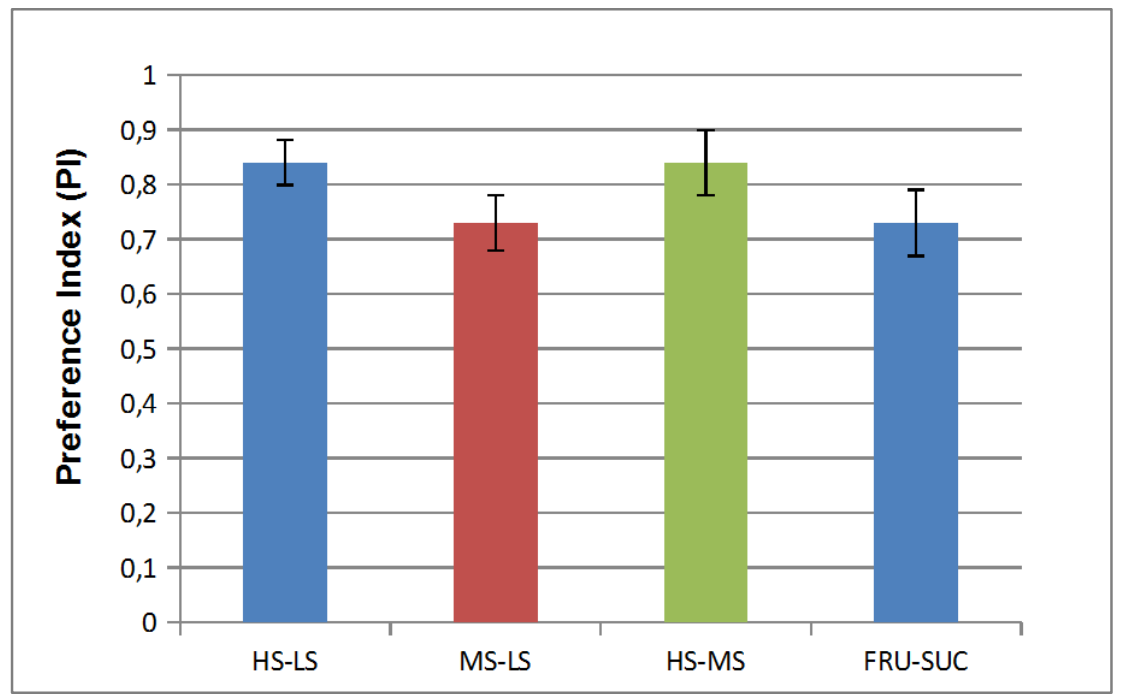

Figure 4. Preference Index (PI) of B. germanica exposed to pairs of fructose solutions of different concentration and one choice of fructose vs sucrose of the same concentration $(\mathrm{HS}=2 \mathrm{~g} / \mathrm{ml} ; \mathrm{MS}=0.2 \mathrm{~g} / \mathrm{ml} ; \mathrm{LS}=0.02 \mathrm{~g} / \mathrm{ml} ; \mathrm{FRU}-\mathrm{SUC}=$ fructose $2 \mathrm{~g} / \mathrm{ml} \mathrm{vS}$ sucrose $2 \mathrm{~g} / \mathrm{ml}$ ). PIs near 1 indicate preference for the most concentrated solution of the pair and for fructose over sucrose

To determine the role of chemoreceptors in the evaluation of food quality, feeding parameters were determined offering $2 \mathrm{~g} / \mathrm{ml}$ sucrose solution as a food source to $\mathrm{B}$. germanica males treated in their mouthparts with $\mathrm{N}$ Ethylmaleimide (NEM), a general blocker of chemoreception. Latency increased significantly in the presence of NEM ( $\mathrm{p}<0.05$, Kruskall-Wallis and Dunn's test; Figure 5a). Conversely, the number of visits and the weight gain were significantly lower in treated insects ( $p<0.05$, Kruskall-Wallis and Dunn's test; Figures $5 b$ and $5 c$ ). In the same way, insects treated with NEM were not able to differentiate between sucrose and fructose solutions of the same concentrations presented simultaneously (Figure 5d). IP of the treated group was not significantly different from 0.5 , indicating a random choice of both sugar sources ( $\mathrm{p}>0.05$, Student's $\mathrm{t}$ test for a parameter). 

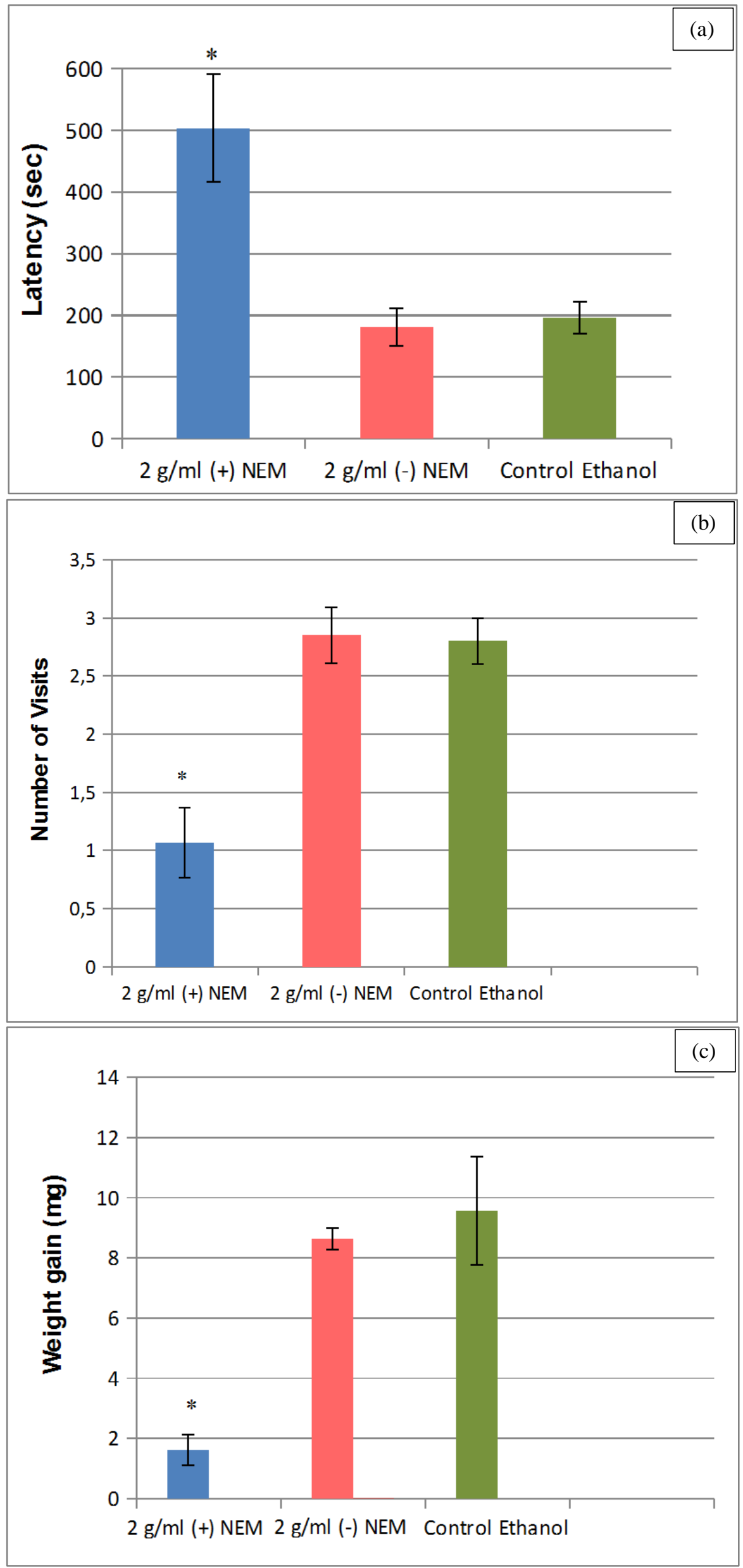


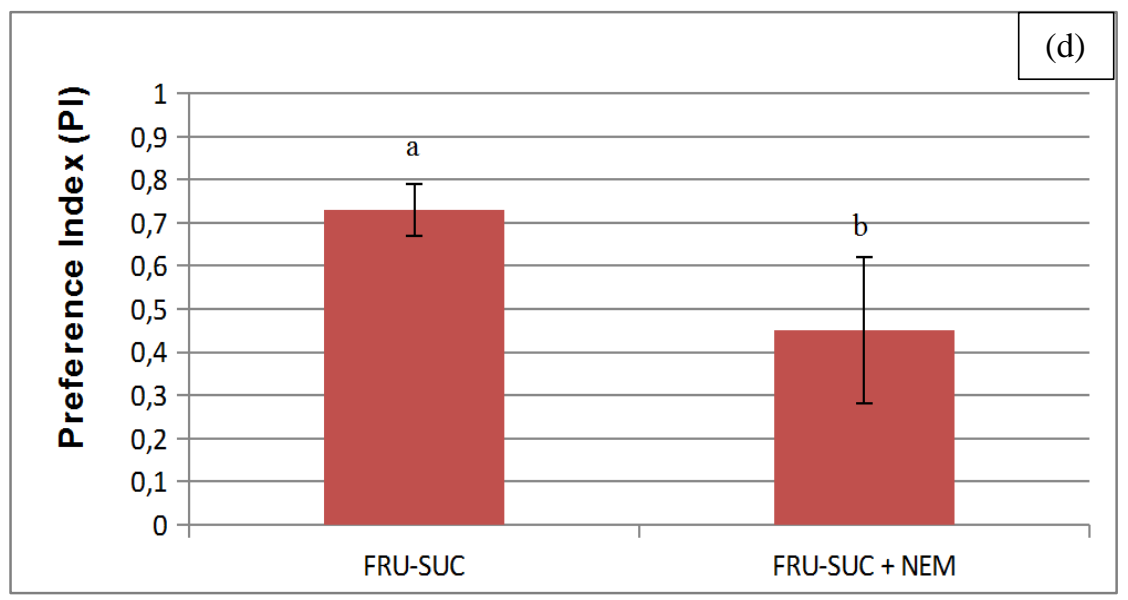

Figure 5. Feeding parameters and sugar preference of B. germanica after treatment with N-Ethylmaleimide (NEM). a) Latency; b) Number of visits to the food source; c) Weight gain; d) Preference Index (PI) for fructose vs sucrose. Marks and different letters indicate statistically significant differences among treatments.

\section{4- Discussion}

In this work we demonstrated that German cockroaches are attracted to food odors, eliciting an orientation behavior towards the food source. This was evidenced by the observation made in short time experiments, in which cockroaches produced high antennal movements and reach the source in few minutes. It was also observed that insects were oriented faster to diets emitting higher amount of volatiles. We also found that cockroaches make a careful taste evaluation before exploiting a food source, being able to distinguish among different concentrations of a simple diet composed only by sugar, and choosing always the most concentrated one. They showed preference for fructose over sucrose at the same concentration, and this capacity was interrupted when mouthparts were treated with an inhibitor of chemoreception.

The use of olfaction and taste to orient and choose a food source is conserved among insects' species, despite of their feeding habits. Herbivores such as grasshoppers touch the surface of a leaf with the sensilla present in its palps to evaluate the chemical composition of the plant. Eventually they can make an exploratory bite to expose chemicals from the inside of the plant and make the decision to accept or reject it as a food source [12]. On the other hand, blood-sucking insects identify vertebrate odors emitted by skin and glands to locate the host. Then, they taste the composition of blood by sampling a small amount of it, before they make the final decision of feed on the host or not [2] [11].

Although many aspects of feeding have been studied in B. germanica, the information regarding the use of chemical signals to locate food is scarce. It was observed that cockroaches oriented towards food emitting odors, and this orientation was enhanced by the presence of feeding conspecifics [13]. The authors concluded that foraging cockroaches probably emit chemical signals associated to feeding. Other mechanisms of orientation towards a food source in B. germanica have been studied by Durier and Rivault [14] [15], who described the mechanism of orientation back to the shelter, after foraging on a food source. They found that the distance from the refuge determined the preference to a source. More recently, it has been observed that the distance from the shelter affects the preference for diets with different carbohydrates and protein ratio, in relation to the energy required to reach the food source [16].

We found that among solid diets, peanut butter showed lower latency, indicating that insects found it faster than other food sources. This food composed by peanut and honey has a high content of carbohydrates and lipids, and emits different volatile compounds, such as aldehyde, ketone, acid, alcohols, etc. [17] [18]. Our observations indicate that these compounds may be used by cockroaches as odor cues to find the source, and are in concordance with other authors that determined that male cockroaches were attracted by head-space extract took from peanut butter in an olfactometer, and identified the active compounds of the extract in an EAG-CG coupled device [19]. When cockroaches were offered with dehydrated yeast, latency was higher compared with peanut butter, but decreased significantly when water was added to the yeast. These observations also suggest that cockroaches orient more efficiently to food depending on the amount of volatiles that it emits, considering that the addition of water to yeast produce compounds of high vapor pressure as secondary metabolites of cellular chemical reactions, such as alcohols, ester, fatty acids, $\mathrm{CO}_{2}$, etc. These compounds can be eventually used as odor cues by cockroaches to start foraging for food [20] [21].

In the case of sucrose solutions, they were detected at higher latencies compared with solid diets. This result was expected due to the low vapor pressure of sugar solutions. However, a significant lower latency was observed in the higher concentration of sucrose tested. More experiments are required to explain this observation.

The role of odors in the velocity of detection of food was confirmed when banana smell was added to the experiment with sugar. A clear decrease in latency was observed in all sucrose solutions in the presence of banana odor. Interestingly, the presence of odors did not modify the amount consumed of each solution, which was directly related 
to their concentration. This point will be further discussed later.

Once an insect gets to a food source, it makes an evaluation of its nutritional quality. In this work we defined two variables that give information about the role of taste and the gustatory preferences of B. germanica. We determined the number of visits to the food source and the weight gain, as a measure of the total amount invested in the experimental time. When cockroaches identify a suitable food source they decide to exploit it, so they forage on the source and exploratory activity decreases significantly. On the other hand, if the food is not convenient, after a short visit to the source, they continue searching for another one. In our experimental context, insects were offered with only one type of food a time, so if they considered it not suitable enough for consumption, they continue searching and the number of visits to the source increased. We observed a lower number of visits to the peanut butter compared with the other solid diets. However, the weight gain was equal to that observed for dehydrated yeast added with water. This result suggests that peanut butter is an attractive diet, considering the low latency, the few visits performed, and the total time spent on the source by cockroaches in the experimental time. However, the relatively low amount ingested at the end of the experiment may be related to the physical traits of the peanut butter, such as viscosity, that reduce the ingestion rate.

The concentration of sugar in a food source is associated to its nutritional quality. Discrimination of concentration using taste receptors helps insects to choose a food source with a high energetic value. Different mechanisms have evolved to help insects to identify nutritious diets. Honey bees associate color patterns present in flowers with its nectar productivity, so they can choose flowers producing high sugar nectar during foraging [22].

We found that German cockroaches also prefer high sugar solutions. The saturated solution of sucrose in distilled water $(2 \mathrm{~g} / \mathrm{ml})$ was the most consumed among all sugar concentrations tested. Moreover, when sugar solutions of different concentration were presented in pairs, cockroaches always preferred the most concentrated one. These results show that B. germanica males make a gustatory evaluation of the sugar source that allows them to choose the more energetically efficient one.

We also found that when a solution of sucrose and one of fructose at equal concentration were presented simultaneously, cockroaches showed preference for fructose, after a few visits to both sources. This result indicates that these insects have the capacity to identify with gustatory evaluations not only the amount of sugar present in a food source, but also its chemical identity. This preference may be related to the presence of specific receptors specialized to different sugar types in the mouthparts, or to the energy provided by the metabolism of each type of sugar. More experiments are required to confirm these hypotheses.

The role of chemoreceptors in the identification of sugars has been confirmed when we applied N-Ethylmaleimide (NEM), a known inhibitor of chemoreception in insects, to cockroaches' mouthparts. This compound has been used in other insect's species to block olfaction as well as taste $[23,24]$. We observed that feeding parameters were modified by the application of NEM. Treated insects showed a higher latency, performed a lower number of visits to the source and the weight gain also decreased. Insects were no longer able to distinguish between sucrose and fructose solutions of the same concentration, feeding equally from both sources. These results suggest that chemoreceptors present in the mandibles, maxilla and palps participate in the general evaluation of food, and particularly in the identification of different sugar types. To confirm this, single-sensillum recordings would be useful to identify the receptors in the mouthparts that are activated by each kind of sugar.

In summary, in this work we described for the first time the feeding behavior of B. germanica associated to the detection of chemical stimuli. We determined how the orientation to a food source is mediated by odors, and the final decision to exploit a food source depends on gustatory evaluations that permit the individual to identify the nutritional value of the source by sensing its concentration. Understanding the feeding behavior of cockroaches may help to enhance control methods, related to the use of toxic gel baits, considering that their effectiveness is strongly associated to their attraction and palatability to guarantee their ingestion.

\section{5- Conclusions}

In this work we studied the role of chemical stimuli in the feeding behavior of B. germanica. The following findings were made:

- Cockroaches find faster the diet that emits volatile compounds, using them as odour cues to locate the food source. Among solid diets, peanut butter was more attractive than the others.

- Regarding the amount ingested, peanut butter was also the more consumed among solid diets, indicating the presence of phagostimulants such as sugars and lipids.

- When sugar solutions were used as food sources, insects locate faster the diets when an odor source was added. The amount ingested increased as concentration of sugar was higher. 
- Insects showed preference for the solution of sugar of higher concentration, when two solutions were presented simultaneously. Moreover, cockroaches preferred fructose over sucrose when solutions of equal concentration of both sugars were presented at the same time.

- This preference was not observed when insects' mouthparts were treated with the general blocker of chemoreception, $\mathrm{N}$-Ethylmaleimide, indicating that cockroaches use chemical stimuli to obtain information from the food source.

- In summary cockroaches use odors emitting from the food source to locate it from a median distance. Then, they taste the diet and make an evaluation of its chemical composition, and choose the food source of higher energetic value.

\section{6- Acknowledgements}

VS is member of the Research Career of the National Council for Scientific and Technological Research from Argentina (Consejo Nacional de Investigaciones Científicas y Técnicas, CONICET).

\section{7- Funding}

The financial support for this work was provided by Chemotecnica S.A. (Buenos Aires, Argentina).

\section{8- Conflict of Interest}

The authors declare no conflict of interest.

\section{9- References}

[1] Kojima, W. "Attraction to carbon dioxide from feeding resources and conspecific neighbours in larvae of the rhinoceros beetle Trypoxylus dichotomus”.PLoS ONE (November 2015). doi: 10.1371/journal.pone.0141733.

[2] Barrozo, R. B. "The Response of the Blood-Sucking Bug Triatoma Infestans to Carbon Dioxide and Other Host Odours." Chemical Senses 29, no. 4 (May 1, 2004): 319-329. doi: 10.1093/chemse/bjh035.

[3] Lauprasert, P., Sitthicharoenchai, D., Thirakhupt, K., and Pradatsudarasar, A. O. "Food Preference and Feeding Behavior of the German Cockroach, Blattella germanica (Linnaeus)" Journal of Scientific Research Chula University, Vol. 31, No. 2 (October 2006): 121-126.

[4] Sugawara, R., S. Kurihara, and T. Muto. "Attraction of the German Cockroach to Cyclohexyl Alkanoates and n-Alkyl Cyclohexaneacetates.” Journal of Insect Physiology 21, no. 5 (May 1975): 957-964. doi:10.1016/0022-1910(75)90107-9.

[5] Wileyto, Paul E., and Mallory G. Boush. "Attraction of the German Cockroach, Blattella Germanica (Orthoptera: Blatellidae), to Some Volatile Food Components." Journal of Economic Entomology 76, no. 4 (August 1, 1983): $752-756$. doi: 10.1093/jee/76.4.752.

[6] Silverman, J., and Bieman, D. N. "Glucose aversion in the German cockroach Blattella germanica”. Journal of Insect Physiology, Vol. 39, No. 11 (November 1993): 925-933.

[7] Wada-Katsumata, A., Silverman, J., and Schal, C. "Differential Inputs from Chemosensory Appendages Mediate Feeding Responses to Glucose in Wild-Type and Glucose-Averse German Cockroaches, Blattella germanica" Chemical Senses, Vol. 36, No 7 (September 2011). doi: 10.1093/chemse/bjr023.

[8] Wada-Katsumata, A., J. Silverman, and C. Schal. "Changes in Taste Neurons Support the Emergence of an Adaptive Behavior in Cockroaches.” Science 340, no. 6135 (May 23, 2013): 972-975. doi: 10.1126/science. 1234854.

[9] Fore, Sara P., H. P. Dupuy, and J. I. Wadsworth. "Correlation of Volatile Components of Peanut Products with Flavor Score I. Shelf Life Studies on Peanut Butter1.” Peanut Science 3, no. 2 (July 1976): 86-89. doi:10.3146/10095-3679-3-2-9.

[10] Manyi-Loh, C.E., Ndip, R.N., and Clarke, A.M. "Volatile Compounds in Honey: A Review on Their Involvement in Aroma, botanical Origin Determination and Potential Biomedical Activities". International Journal of Molecular Science, Vol. 12 (December 2011): 9514-9532. doi: 10.3390/ijms12129514.

[11] Friend, W G, and J J B Smith. "Factors Affecting Feeding by Bloodsucking Insects.” Annual Review of Entomology 22, no. 1 (January 1977): 309-331. doi: 10.1146/annurev.en.22.010177.001521.

[12] Chapman, R. F. "The insects. Structure and function”. Edited by Simpson, S. J and Douglas, A. E., Fifth Edition (1998). Cambridge University Press, 929 pp.

[13] Lihoreau, M. and Rivault, C. "Local enhancement promotes cockroach feeding aggregations" PLoS ONE 6(7) (July 2011): e22048. doi: 10.1371/journal.pone.0022048. 
[14] Durier, Virginie, and Colette Rivault. "Path Integration in Cockroach larvae,Blattella Germanica (L.) (insect: Dictyoptera): Direction and Distance Estimation.” Animal Learning \& Behavior 27, no. 1 (March 1999): 108-118. doi:10.3758/bf03199436.

[15] Durier, Virginie, and Colette Rivault. "Effects of Spatial Knowledge and Feeding Experience on Foraging Choices in German Cockroaches.” Animal Behaviour 62, no. 4 (October 2001): 681-688. doi:10.1006/anbe.2001.1807.

[16] Ko, A. E., Jensen, K., Schal, C., and Silverman, J. "Effects of foraging distance on macronutrient balancing and performance in the German cockroach Blattella germanica. Journal of Experimental Biology, Vol. 220 (2017): 304-3011. doi: $10.1242 /$ jeb. 146829 .

[17] Young, Clyde T., and Allan R. Hovis. "A Method for the Rapid Analysis of Headspace Volatiles of Raw and Roasted Peanuts.” Journal of Food Science 55, no. 1 (January 1990): 279-280. doi: 10.1111/j.1365-2621.1990.tb06078.x.

[18] Chestchik, I., Granvogl, M., and Schieberle, P. "Quantitation of key peanut aroma compounds in raw peanut and pan-roasted peanut meal. Aroma reconstitution and comparison with commercial peanut products”. Journal of Agricultural Food and Chemistry", Vol. 58, No 20 (2010): 11018-11026. doi: 10.1021/jf1026636.

[19] Karimifar, N., Gries, R., Khaskin, G., and Gries, G. "General food semiochemicals attract ominvorous German cockroaches, Blattella germanica". Journal of Agricultural and Food Chemistry, Vol. 59, No 4 (January 2011): 1330-1337. doi: $10.1021 / \mathrm{jf} 103621 \mathrm{x}$.

[20] Saerens, S. M. G., F. Delvaux, K. J. Verstrepen, P. Van Dijck, J. M. Thevelein, and F. R. Delvaux. "Parameters Affecting Ethyl Ester Production by Saccharomyces Cerevisiae During Fermentation.” Applied and Environmental Microbiology 74, no. 2 (November 9, 2007): 454-461. doi: 10.1128/aem.01616-07.

[21] Eder, M., Sánchez, I., Brice, C., Camarassa, C., Legras, J-L., and Dequin, S. "QTL mapping of volatile compound production in Saccharomyces cerevisiae during alcoholic fermentation” BMC Genomics, Vol. 19 (March 2018): 166. doi: 10.1186/s12864-018-4562-8.

[22] Giurfa, M., Núñez, J. A., Chittka, L., Menzel, R. "Color preferences of flower-naive honeybees”. Journal of Comparative Physiology A (Sensory, Neural, Behavioral Physiology), Vol. 177, No 3 (January 1995): 247-259. doi: 10.1007/BF00192415.

[23] Alzogaray, R. A., A. Fontan, and E. N. Zerba. "Repellency of Deet to Nymphs of Triatoma Infestans." Medical and Veterinary Entomology 14, no. 1 (March 2000): 6-10. doi: 10.1046/j.1365-2915.2000.00213.x.

[24] González, P. V., González-Audino, P. A., and Masuh, H. M. "Behavioral Response of Aedes aegypti (Diptera: Culicidae) Larvae to Synthetic and Natural Attractants and Repellents" Journal of Medical Entomology, Vol. 52, No 6, (November 2015): 1315321. doi: 10.1093/jme/tjv136. 